\title{
N-Body Simulations of Planetesimal Evolution: Effect of Varying Impactor Mass Ratio
}

\author{
Zoë M. Leinhardt and Derek C. Richardson \\ Department of Astronomy, University of Maryland, College Park, Maryland 20742-2421 \\ E-mail: zoe@astro.umd.edu
}

Received September 14, 2001; revised March 14, 2002

We present results from direct $N$-body simulations of collisions between gravitational aggregates of varying size as part of a study to parameterize planetesimal growth in the Solar System. We find that as the ratio of projectile to target mass departs from unity, the impact angle has less effect on the outcome. At the same time, the probability of planetesimal growth increases. Conversely, for a fixed impact energy, collisions between impactors with mass ratio near unity are more dispersive than those with impactor mass ratio far from unity. We derive an expression for the accretion probability as a function of mass ratio. For an average mass ratio of $1: 5$, we find an accretion probability of $\sim 60 \%$ over all impact parameters. We also compute the critical specific dispersal energy $Q_{D}^{*}$ as a function of projectile size. Extrapolating to a projectile size of $1 \mathrm{~m}$ with a 1-km target, we find $Q_{D}^{*}=10^{3}-10^{4} \mathrm{~J} \mathrm{~kg}^{-1}$, in agreement with several other collision models that use fundamentally different techniques. Our model assumes that the components of each gravitational aggregate are identical and indestructible over the range of sampled impact speeds. In future work we hope to incorporate a simple fracture model to extend the range of applicable speeds and we plan to implement our results in a large-scale planetesimal evolution code. (c) 2002 Elsevier Science (USA)

Key Words: planetesimals; asteroids; planetary formation; collisional physics.

\section{INTRODUCTION}

This paper is part of a larger project to investigate planetesimal evolution in the context of Solar System formation (Leinhardt et al. 2000). In order to create an accurate numerical model of Solar System formation it is necessary to understand how the planetary building blocks, namely, kilometer-sized planetesimals, evolve and grow into larger bodies. In the research presented here we find conditions necessary for planetesimal growth. Our goal is to provide a recipe for planetesimal evolution that can be used in Solar System formation models.

Over the past decade evidence has been mounting that small bodies several hundreds of meters to tens of kilometers in size are gravitational aggregates (Leinhardt et al. 2000; Richardson et al. 2002 and references therein). Accordingly, we model our planetesimals as 0.25 to $1 \mathrm{~km}$ rubble piles-gravitational aggregates with no tensile strength (Richardson et al. 2002). We assume that planetesimal evolution in the early Solar System is dominated by slow (a few $\mathrm{m} \mathrm{s}^{-1}$ ) orbit-crossing collisions between planetesimals. Thus, our simulations focus on slow collisions between rubble-pile planetesimals.

In this paper the study of planetesimal evolution is split into two experiments. In the first experiment we quantify which collisions cause planetesimal growth or erosion (Sections 3.1 and 3.2). We consider collisions between planetesimals of different masses over a range of impact parameters and speeds. We derive accretion/erosion probabilities on the basis of this experiment (Section 3.3). In the second experiment we determine the critical dispersal energy $\left(Q_{D}^{*}\right)^{1}$ as a function of the mass ratio of the larger rubble pile to the smaller rubble pile (Sections 4.1 and 4.2). This allows us to compare our results directly with those of other groups (Section 4.3).

It is useful to define accretion and erosion as they pertain to this paper. Accretion is the permanent retention of new mass, whereas erosion is the permanent loss of mass. In our simulations the largest initial rubble pile is said to have accreted material if it has gained mass at the end of the simulation and to have eroded if it has lost mass. A simulation is ended when the collision event has terminated. In our simulations termination of the collision event is reached when less than $10 \%$ of the system mass is accreting or orbiting the largest postcollision remnant (Section 2.2).

The remainder of this paper is divided into three sections. In Section 2 we summarize our numerical method. In Sections 3 and 4 we present the results of the accretion/erosion and critical dispersal simulations, respectively, and we compare these results to previous experiments. In Section 5 we discuss the limitations of our method and plans for future work.

\section{METHOD}

A detailed description of the numerical method used in these simulations is given in Leinhardt et al. (2000). In this section

\footnotetext{
${ }^{1} Q_{D}^{*}$ is the energy per unit mass necessary to create a postcollision remnant of $50 \%$ the mass of the system (see Section 4.1 ).
} 

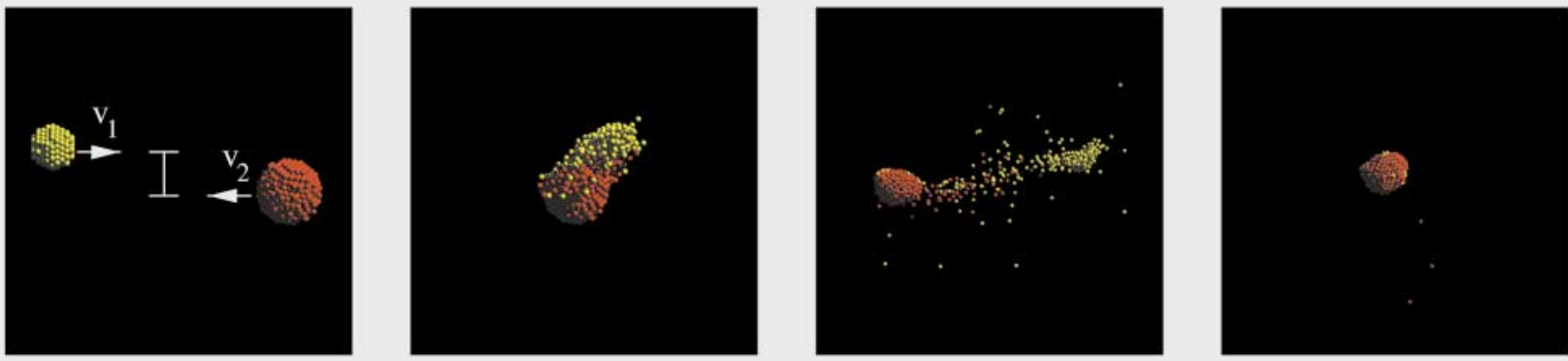

FIG. 1. Visualization of a simulation. The yellow object is one third the mass of the red object. The impact parameter in this example is 0.75 and the initial relative speed is $1 v_{\text {crit }}=v_{2}+v_{1}$.

we present a summary of the numerical method and identify differences in the methodology.

\subsection{Planetesimal Model}

Typical initial conditions used in the collision simulations are illustrated in Fig. 1. A Cartesian coordinate system is used with the origin at the center of mass. Initially, each simulation begins with two rubble piles set 2.5 Roche radii apart (in the $\pm x$ direction) to ensure that tidal forces are small. In most simulations presented here one rubble pile is significantly smaller than the other (less than half the mass of the larger rubble pile). In these cases we consider the smaller rubble pile the projectile (yellow rubble pile in Fig. 1) and the larger rubble pile the target (red rubble pile in Fig. 1). However, it should be noted that, unlike in laboratory collision experiments, the projectile is a significant fraction of the target's mass. We define the mass ratio as $M_{P} / M_{T}$, that of the mass of the projectile to the mass of the target. In all cases presented here, $M_{P} /$ $M_{T} \leq 1.0$.

Each rubble pile is built with identical spherical particles of $3.5 \mathrm{~g} \mathrm{~cm}^{-3}$ bulk density using a hexagonal close-packed form (Leinhardt et al. 2000). The target has either $\sim 1000$ particles (Sections 3.1 and 3.2) or 2000 particles (Sections 4.1 and 4.2). The projectiles have between 27 and 955 particles depending on the mass ratio ( $1: 64$ to $1: 1)$ and the experiment type (accretion/ erosion (Section 3.1) or critical dispersal (Section 4.1)). Our rubble piles have a packing efficiency of $\sim 55 \%$, yielding a bulk density of $\sim 2 \mathrm{~g} \mathrm{~cm}^{-3}$. The impact parameter $b$ is defined at impact in units of the sum of the radii $R_{P}+R_{T}$, so $b=0$ is a head-on collision and $b=1$ is a glancing collision. Although the trajectories of the projectile and target will be affected by gravitational focusing, for simplicity we assume that trajectory deflection is zero; therefore,

$$
b=\sin \phi,
$$

where $\phi$ is the impact angle in the absence of deflection (between the line of centers and the $x$-component of the line of centers). In the simulations presented here $b$ ranges from 0 to 0.75 . For $b>0.75$, there is little or no mass exchange between the projectile and the target (Leinhardt et al. 2000); thus, we do not investigate scenarios in this regime. Both the projectile and the target are given initial speeds between 1 and $20 \mathrm{~m} \mathrm{~s}^{-1}$ in the direction of the other body (Fig. 1) such that the center of mass is stationary. The speed of the encounter is limited on the low end by the assumption that both objects are initially on hyperbolic orbits. The largest initial speeds are limited in magnitude by requiring that they not greatly exceed the threshold for significant fracturing of rock (Leinhardt et al. 2000).

The collisional behavior of each particle is governed by normal and tangential coefficients of restitution, $\epsilon_{n}$ and $\epsilon_{t}$, respectively. For most particle collisions $\epsilon_{n}$ is set to 0.8 , which allows dissipation during a collision, and there is no surface friction; i.e., $\epsilon_{t}=1.0$. However, if the relative speed of two colliding particles is less than $10 \%$ of their mutual escape speed, $\epsilon_{n}$ is set to unity to prevent excessive bouncing (Richardson 1994).

In Leinhardt et al. (2000) collision outcome as a function of impactor spin was explored. It was found that oppositely oriented spins reduced mass dispersal in general, while aligned spins, depending on the orbital angular momentum, enhanced mass dispersal. In addition, asymmetries introduced by spin momenta often resulted in asymmetric remnant shapes. These effects are not explored in the present study, which concentrates solely on the effect of varying impactor mass ratio. However, we expect that the results would be analogous to the original findings if spin were introduced, though presumably the smaller the projectile, the less effect its spin would have on the outcome. Also, an experiment varying $\epsilon_{n}$ was performed in Leinhardt et al. (2000), with the result that smaller values of $\epsilon_{n}$ (greater dissipation) gave rise to larger, more numerous, reaccreted remnants. Similarly, we would expect smaller values of $\epsilon_{n}$ to enhance remnant production in the present study, but we do not explore this here. We note that since energy dissipation in an inelastic collision goes as $\sim 1-\epsilon_{n}^{2}$, the effective binding energy could be adjusted by a similar factor to take into account a different dissipation parameter. Testing this possibility is deferred to future work. 


\subsection{Numerical Code}

Our simulations were performed using a modified version of the cosmological $N$-body code pkdgrav, which uses a loworder leap-frog integrator (see Richardson et al. (2000) and Leinhardt et al. (2000) for details). In our implementation of pkdgrav, inelastic bouncing is the only allowed outcome of particle collisions; there is no merging or fracturing of particles.

The run time for our simulations was initially about five times the free-fall time,

$$
t_{f} \sim \sqrt{\frac{x^{3}}{G M}}
$$

where $x$ is the initial separation of the rubble piles along the $x$-axis, $M$ is the combined mass $M_{P}+M_{T}$, and $G$ is the gravitational constant. Typically, $t_{f} \sim 40 \mathrm{~h}$. In most cases this is sufficient time for the postcollision system to reach steady state. Simulations are run longer (by a factor of 2 to 4 ) if the mass accreting onto and/or orbiting the largest postcollision remnant is greater than $10 \%$ of the total mass of the system.

The time step for each run was set to $t_{0} \sim 50 \mathrm{~s}\left(\simeq 10^{-5}\right.$ year $\left./ 2 \pi\right)$ times a speed-dependent scaling factor $1 /(2 v+1)$, where $v$ is in units of $v_{\text {crit }}$, a convenient measure (Leinhardt et al. 2000) found by equating the initial total kinetic energy to the binding energy of a rubble pile made up of a homogeneous mixture of both the projectile and the target:

$$
v_{\text {crit }}=M \sqrt{\frac{6 G}{5 \mu R}} .
$$

Here $\mu$ is the reduced mass $M_{P} M_{T} / M$, and $R$ is the radius of a sphere of mass $M$, assuming the same bulk density:

$$
R=\left(R_{P}^{3}+R_{T}^{3}\right)^{1 / 3} .
$$

The scaling term results in smaller time steps for simulations at higher speed, which reduces the chance of missing a collision between particles that would otherwise result in an error condition. Since $v$ is of order unity, $t_{0}$ is about two orders of magnitude smaller than the dynamical time $\sim 1 / \sqrt{G \bar{\rho}} \sim 1 \mathrm{~h}$ for an object with a bulk density $\bar{\rho} \sim 2 \mathrm{~g} \mathrm{~cm}^{-3}$. We have chosen an output frequency of 200 outputs per simulation in order to produce enough data for analysis without taking up an impractical amount of disk space.

\subsection{Hardware}

Most of the simulations were run on a local Beowulf cluster consisting of 24 machines with 1-GHz Athlon CPUs using the High Throughput Computing environment condor (see Leinhardt et al. 2000; http://www.cs.wisc.edu/ condor) under RedHat Linux 7.1. One set of simulations was run on a Beowulf cluster of 32 machines with 1.2-GHz Athlon CPUs at the University of California, Santa Cruz.

\section{ACCRETION/EROSION SIMULATIONS}

\subsection{Accretion/Erosion: Method}

In the first experiment we conducted four parameter-space studies, each with a different mass ratio (1:1 from Leinhardt et al. 2000, 1:3, 1:6, and 1:9). In all of these studies the target had a mass of $8 \times 10^{12} \mathrm{~kg}$, a radius of $\sim 1 \mathrm{~km}$, and contained 955 particles. For each mass ratio we explored the parameter space of $b$ and $v$ (impact parameter and speed, respectively) near the transition between accretion and erosion. The range of $b$ for each study was from 0 to 0.75 in steps of 0.15 . The range of velocity changed from study to study in order to follow the accretion/erosion transition (Fig. 2), which depends on the mass ratio. The initial speeds ranged from 2.1 to $3.4 \mathrm{~m} \mathrm{~s}^{-1}$ (1.00 to $1.60 v_{\text {crit }}$ ), 2.5 to $3.8 \mathrm{~m} \mathrm{~s}^{-1}$ (1.00 to $1.50 v_{\text {crit }}$ ), and 2.8 to $3.6 \mathrm{~m} \mathrm{~s}^{-1}$ ( 0.90 to $\left.1.30 v_{\text {crit }}\right)$ in steps of 0.10 for mass ratios $1: 3,1: 6$, and $1: 9$, respectively.

For each mass ratio we ran between 24 and 28 simulations to resolve the transition between accretion and erosion. The transition was deemed resolved at a given $b$ if there was at least one simulation that resulted in erosion and one simulation that resulted in accretion. The collision speed at the transition, $V_{\text {trans }}$, was determined by a linear interpolation between the minimum collision speed that resulted in erosion and the maximum collision speed that resulted in accretion.

\subsection{Accretion/Erosion: Results}

Figure 2 summarizes the results of the accretion/erosion simulations (Fig. 1 gives snapshots of one simulation). Each grid shows the parameter space explored in $b$ and $v$ for a given mass ratio. The shape traced in each box is the cross section of the largest post-collision remnant along its longest axis. The objects in boxes bounded with dashed lines have been eroded as a result of the collision. In other words, the largest post-collision remnant has a mass that is less than the original target. The objects in the boxes bounded with solid lines have accreted mass. The largest post-collision remnant has a mass larger than the original target. The accretion/erosion curve $v_{*}(b)$ is the function that describes the transition between the erosion and accretion events. In order to resolve the accretion/erosion curve more clearly, $V_{\text {trans }}$ was determined using the method described in Section 3.1 at each $b$ for each mass ratio. $V_{\text {trans }}$ is shown in Fig. 3 along with fits for the accretion/erosion curve. The error bars, which are half of the difference between the speed of the simulation above the transition and the speed below the transition, approximate the error in the linear interpolation used to find $V_{\text {trans }}$. The fit for the accretion/erosion curve for mass ratio $1: 1$ (Fig. 3 ) is a Gaussian,

$$
v_{*}(b ; \xi)=\alpha \exp \left[-\frac{(b-\beta)^{2}}{\gamma}\right]+\delta,
$$

where $\xi=M_{P} / M_{T}$ is the mass ratio (1:1 in this case), and $\alpha, \beta, \gamma$, and $\delta$ are determined by a nonlinear least-squares fit (Leinhardt et al. 2000). Mass ratios $1: 3,1: 6$, and $1: 9$ are well 

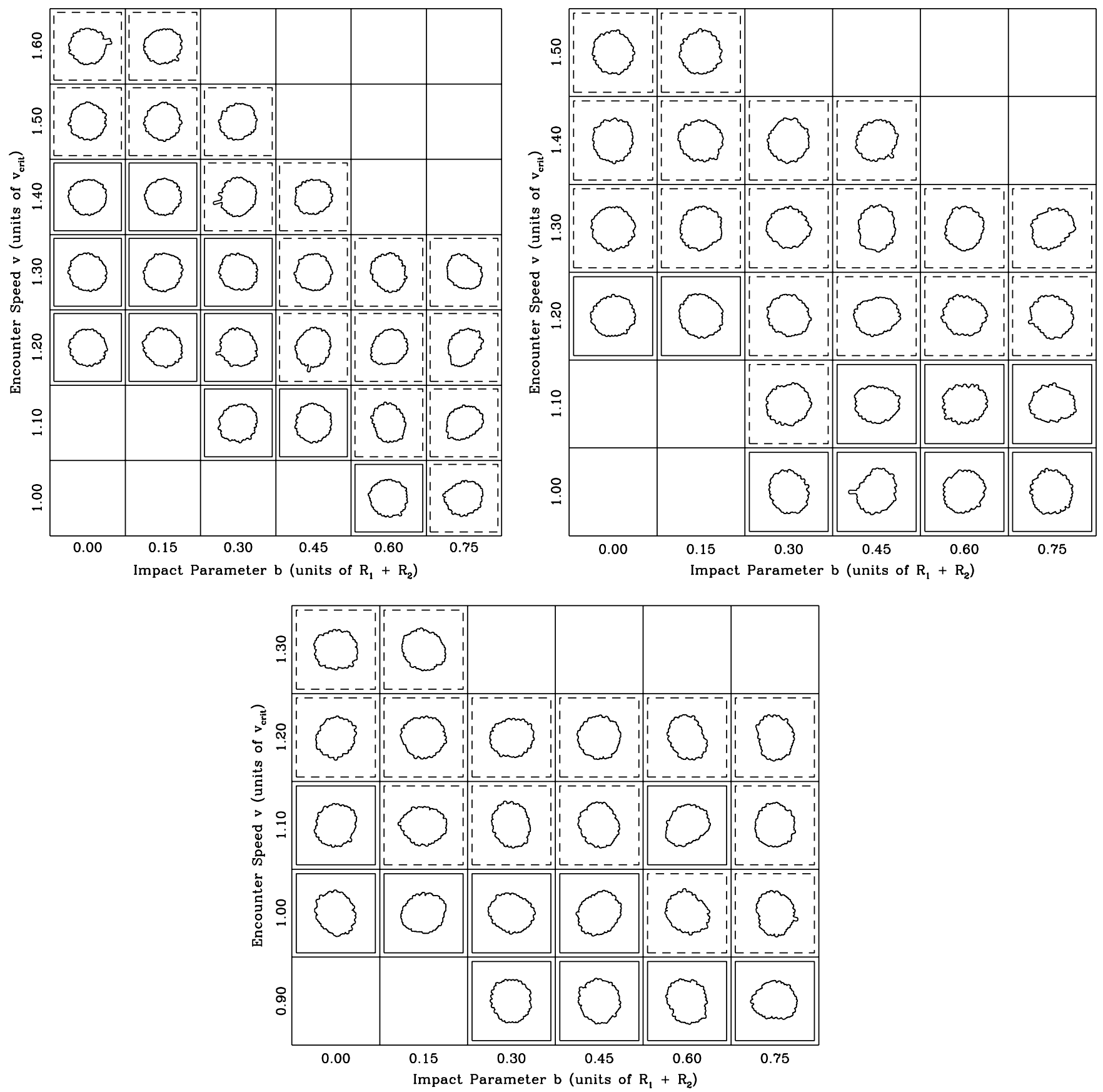

FIG. 2. Parameter space of accretion/erosion simulations. The mass ratios are $1: 3,1: 6$, and $1: 9$ for the grids shown at the top left, top right, and bottom, respectively. The results of the $1: 1$ simulations are shown in Leinhardt et al. (2000). The $x$-axes are impact parameter $b$ in units of the sum of the radii. The $y$-axes are speed in units of $v_{\text {crit }}$. Each grid box filled with a cross section represents one simulation. The cross section is a slice through the largest post-collision remnant along its longest axis. The boxes with a dashed outline are erosion events. Those with solid lines are accretion events. The transition between them is the accretion/erosion curve.

characterized by progressively shallower linear functions,

$$
v_{*}\left(b ; \xi_{i}\right)=m_{i} b+c_{i},
$$

where $\xi_{i}=1: 3,1: 6,1: 9$, and the slope and intercept, $m_{i}$ and $c_{i}$ respectively, are determined using a weighted linear leastsquares fit. From Fig. 3 it is clear that as the mass ratio departs from unity, $b$ becomes less and less important to the collision outcome.

\subsection{Accretion/Erosion: Discussion}

Given the data presented above we can calculate the probability that for a given mass ratio a collision will result in growth 


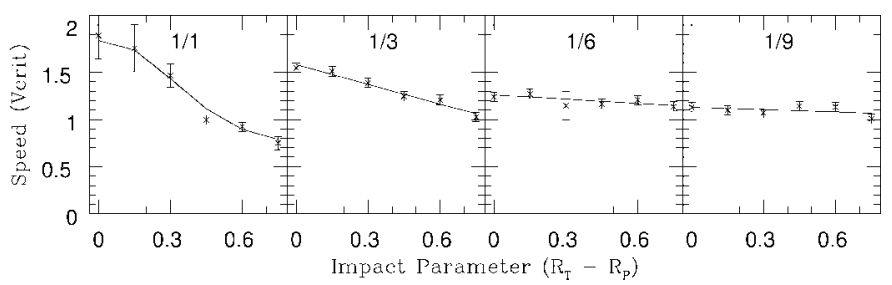

FIG. 3. The accretion/erosion curve plotted for four different mass ratios. The $y$-axis is speed in units of $v_{\text {crit }}$. The $x$-axis is impact parameter in units of the sum of the radii of the impactor and the projectile. The accretion/erosion curve on the far left (mass ratio of $1: 1$ ) was fit by a Gaussian (Leinhardt et al. 2000). All other mass ratios $(1: 3,1: 6$, and $1: 9)$ were fit with linear functions: the slopes are $-0.79 \pm 0.08,-0.14 \pm 0.08$, and $-0.08 \pm 0.08$, respectively.

of the target. In order to do this we need to assume both an impact parameter and velocity distribution. If we assume that the velocity distribution is Maxwellian with $v_{r m s}$ equaling the escape velocity $v_{e}$ from the target and that the impact parameter distribution is uniform, the probability of planetesimal growth from a collision is

$$
P\left[f(b, v) \geq \frac{M_{T}}{M}\right]=\frac{1}{\pi \int_{0}^{1} b d b} \pi \int_{0}^{1} b d b \frac{\int_{v_{0}(b)}^{v_{*}(b)} g(v) d v}{\int_{v_{0}(b)}^{\infty} g(v) d v}
$$

where $f(b, v)$ is the mass fraction of the largest postcollision remnant, $v_{*}(b)$ is the critical dispersal fit described above (we have dropped the $\xi_{i}$ to simplify the equation), $g(v)$ is the normalized Maxwellian distribution of relative speed,

$$
g(v) d v=\frac{1}{2 \sqrt{\pi} v_{e}^{3}} \exp \left(-\frac{v^{2}}{4 v_{e}^{2}}\right) v^{2} d v
$$

and $v_{0}(b)$ is the minimum initial speed, in units of $v_{\text {crit }}$, for a hyperbolic encounter $\left(v_{\infty}>0\right)$. The expression for the speed at infinity is

$$
v_{\infty}=\left(v^{2}-\frac{2 G M \cos \phi}{x v_{\text {crit }}}\right)^{\frac{1}{2}}
$$

where the second term is due to gravitational focusing ( $x$ is the initial separation along the $x$-axis; cf. Eq. (2)). If $v_{\infty}=0$, then $v=v_{0}$ and

$$
v_{0}=\sqrt{\frac{2 G M \cos \phi}{x v_{\text {crit }}^{2}}}
$$

Substituting for $\phi$ from Eq. (1), we find $v_{0}$ as a function of $b$ :

$$
v_{0}(b)=\sqrt{\frac{2 G M \sqrt{1-b^{2}}}{x v_{\text {crit }}^{2}}} .
$$

From our simulations, we find the probability that a collision between two rubble-pile planetesimals will result in the growth of one of the planetesimals is $37 \pm 3,46 \pm 1,73 \pm 1$, and $76 \pm 1 \%$ for mass ratios of $1: 1,1: 3,1: 6$, and $1: 9$, respectively. Figure 4 shows the probability of an accretion event as a function of mass ratio fit with a power law of slope $-0.47 \pm 0.05$.

Next we find the probability of an accretion event for the mean mass ratio,

$$
\bar{\xi}=\frac{\int_{\xi_{1}}^{\xi_{2}} \xi \eta(\xi) d \xi}{\int_{\xi_{1}}^{\xi_{2}} \eta(\xi) d \xi}
$$

where $\xi_{1}$ is the mass ratio with the largest difference in mass between projectile and target, $\xi_{2}$ is the mass ratio with the smallest difference in mass, and $\eta(\xi)$ is the distribution of $\xi$. We assume that the planetesimals have a power law distribution of size

$$
d N \propto R^{-\alpha} d R
$$

where $N$ is the number of planetesimals, $R$ is the radius of a planetesimal, and $\alpha$ is the power law index. Assuming constant bulk density, we can express $d N$ in terms of mass,

$$
d N \propto M^{-(\alpha+2) / 3} d M .
$$

The mass ratio distribution function then has the same form,

$$
\eta(\xi)=\xi^{-(\alpha+2) / 3}
$$

Since $\eta(\xi)$ is a power law, it will diverge as $\xi$ approaches zero

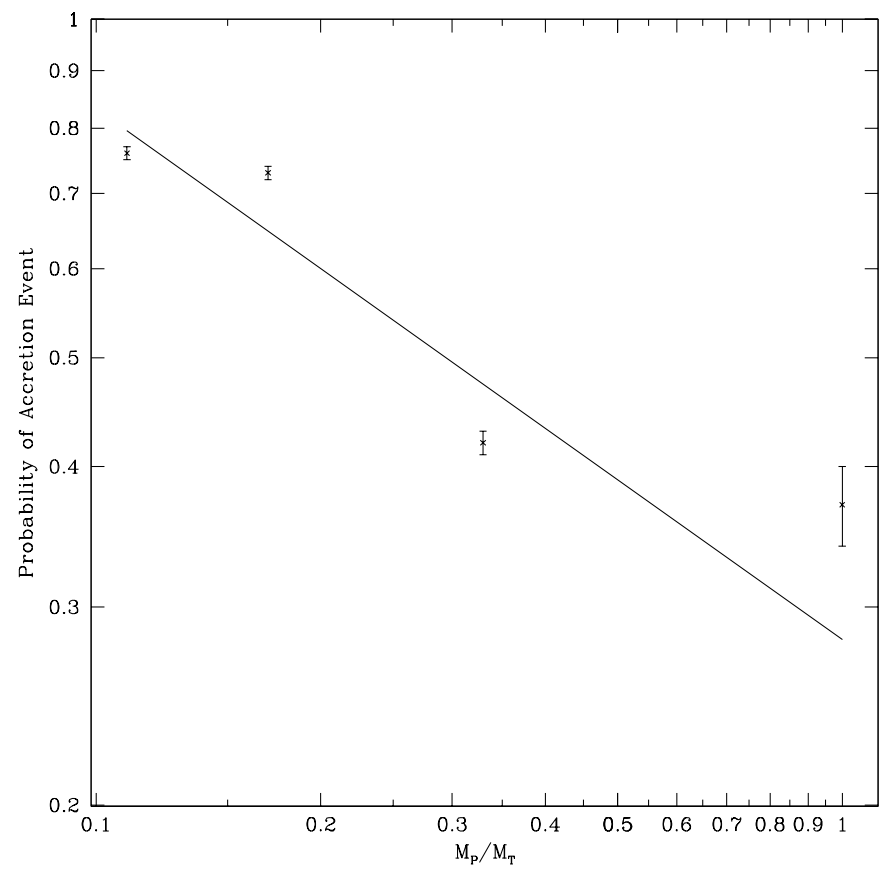

FIG. 4. Probability of an accretion event as a function of mass fraction. The solid line is a power-law fit to the data with a slope of $-0.47 \pm 0.05$ and an intercept of $-0.55 \pm 0.04$. The error bars represent the error in the $v_{*}(b)$ fits. 

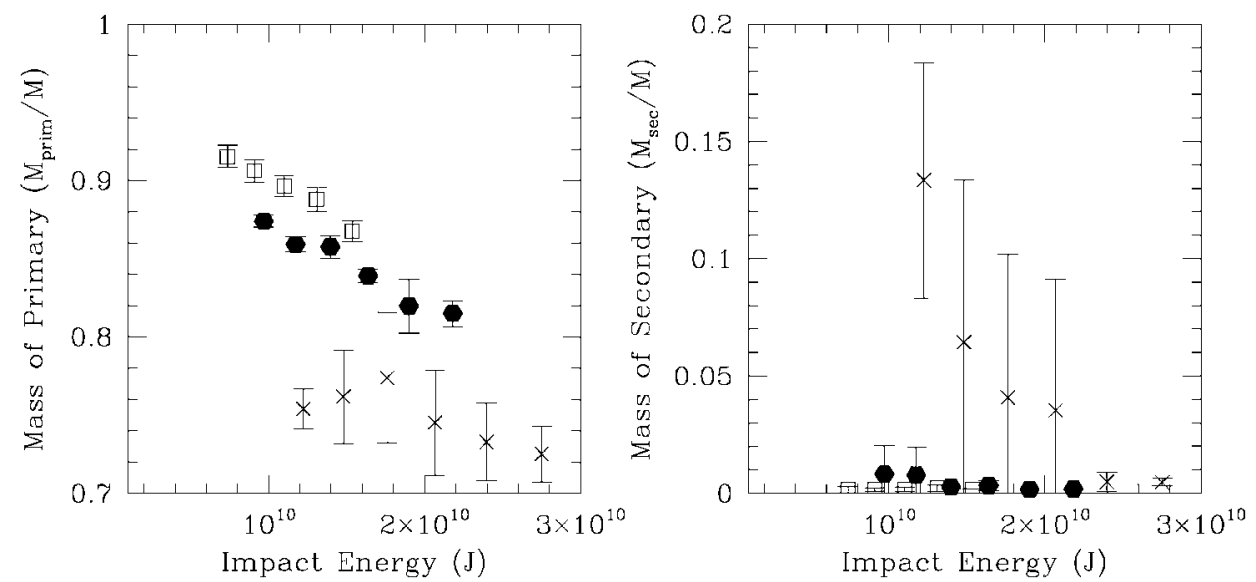

FIG. 5. Plots of the largest and second-largest post-collision remnants as a function of impact energy. The crosses, filled hexagons, and open squares are from mass ratios $1: 3,1: 6$, and $1: 9$, respectively. All data points are averaged over $b$; the error bars are the rms.

(that is, as the mass ratio gets large); thus we define $\xi_{1}$ to be the mass ratio where the probability of an accretion event is unity. From the fit in Fig. 4, $\xi_{1} \sim 0.06$. The upper limit $\xi=1$ since $M_{P} \leq M_{T}$. If we take $\alpha=3$, then $\bar{\xi}=0.22$ or $\sim 1: 5$. From Fig. 4 the probability of an accretion event for $M_{P} / M_{T}=\bar{\xi}$ is then $\sim 57 \%$, which means on average the target will grow. The more interesting question is how does the mean mass ratio and its corresponding probability for an accretion event change with time. This is complicated because the population changes after each collision, which means that $v_{e}$ and $v_{\text {rms }}$ will also eventually change. This coupling suggests that a numerical approach is needed to determine the evolution, a project we defer to future work.

It is also interesting to examine how the size of the projectile affects the efficiency of collision. Figure 5 shows the mass of the largest (primary) and second-largest (secondary) post-collision remnant as a function of impact energy,

$$
E=\frac{\mu v^{2}}{2}
$$

where $v$ is the relative speed in $\mathrm{m} \mathrm{s}^{-1}$. To identify the primary and secondary, we used the clump-finding algorithm described in Leinhardt et al. (2000). Notice that for the same impact energy the mass of the primary from the $1: 3$ simulations (crosses) is less than that for $1: 6$ (filled hexagons), which is less than that for $1: 9$ (open squares). In addition, the secondary from the $1: 3$ simulations is significantly larger than the secondaries from the $1: 6$ and $1: 9$ simulations. These results show that for a given impact energy, a larger projectile is more efficient at disrupting the target than a smaller projectile. Basically, the larger projectile hits more particles but imparts less energy to them than a smaller projectile. Similar results were found by Benz and Asphaug (1999) and Benz (2000). In addition, the mass distribution of the post-collision remnants is more shallow for a large projectile than for a small projectile.

\section{CRITICAL DISPERSAL SIMULATIONS}

\subsection{Critical Dispersal: Method}

In this experiment we computed the critical dispersal energy $\left(Q_{D}^{*}\right)$ as a function of impactor mass ratio. $Q_{D}^{*}$ is defined as the minimum kinetic energy per unit total mass necessary to create a postcollision remnant equal to $50 \%$ of the mass of the total system while the rest of the mass is dispersed to infinity (Durda et al. 1998). In these simulations we kept the impact parameter fixed at $b=0$ (head-on collision) and ran five massratio models $(1: 8,1: 9,1: 16,1: 32$, and $1: 64)$. Each model was run for at least 10 collision speeds in order to bracket $Q_{D}^{*}$. Because the mass ratios are far from unity in these simulations, we approximately doubled the resolution (number of particles) by using a target of $\sim 2000$ particles. However, the smallest projectiles (1:32 and $1: 64$ the mass of the target) still had relatively few particles and were therefore not very spherical. This meant that the orientation of the projectile had a significant effect on the collision outcome. In order to take this into account each massratio system was run eight times at the same speeds with the projectile in different orientations. The critical dispersal speed $V_{\text {dis }}$ (the speed necessary for critical dispersal) was found using linear interpolation with a method similar to that used to find $V_{\text {trans }}$ (Section 3.1).

\subsection{Critical Dispersal: Results}

Figure 6 shows $V_{\text {dis }}$ for each mass ratio averaged over all orientations. The error bars are the rms of the distribution of $V_{\text {dis }}$ at any given mass ratio. The solid line is a least-squares powerlaw fit with a slope of $-1.9 \pm 0.1$. Figure 7 shows the critical dispersal energy necessary to disperse $50 \%$ of a $1-\mathrm{km}$ target. For this figure we converted the $V_{\text {dis }}$ values to $Q_{D}^{*}$ using

$$
Q_{D}^{*}=\frac{\mu V_{\mathrm{dis}}^{2}}{2 M}
$$


The error bars are propagated from the $V_{\mathrm{dis}}$ data. The fit is a power law of slope $-1.1 \pm 0.3$ and intercept $3.5 \pm 0.7$. For a $1-\mathrm{m}$ projectile this fit gives $Q_{D}^{*} \sim 10^{2.8}-10^{4.2} \mathrm{~J} \mathrm{~kg}^{-1}$, which is consistent with those found by Love and Ahrens (1996) and Benz and Asphaug (1999), for example, but disagrees with that found by Ryan and Melosh (1998).

\subsection{Critical Dispersal: Discussion}

In many respects our simulations were conducted in a similar way to those of Love and Ahrens (1996). Using a 3D smoothed particle hydrocode with a Tillotson equation of state for granite without strength or fracturing, they ran several simulations at various target diameters (10-1000 km) and speeds (3, 5, and $7 \mathrm{~km} \mathrm{~s}^{-1}$ ) to find $Q_{D}^{*}$ as a function of target diameter. For each target size they found $Q_{D}^{*}$ by changing the projectile size and interpolating or extrapolating to find the energy necessary to produce a primary of $50 \%$ the mass of the system. They placed their data on the $Q_{D}^{*}$ vs $D$ plot first constructed by Holsapple (1994) without correction for different projectile sizes. Our results from Section 3.2 suggest that projectile size is important in determining $Q_{D}^{*}$. However, although the projectile size changed by two orders of magnitude, over half of their simulations used a projectile that was $<1 / 100$ the mass of the target-small enough that changes in the projectile size may not be important. Benz and Asphaug (1999) found results similar to those of Love and Ahrens (1996) but with a more sophisticated code that included an explicit model of fracture.

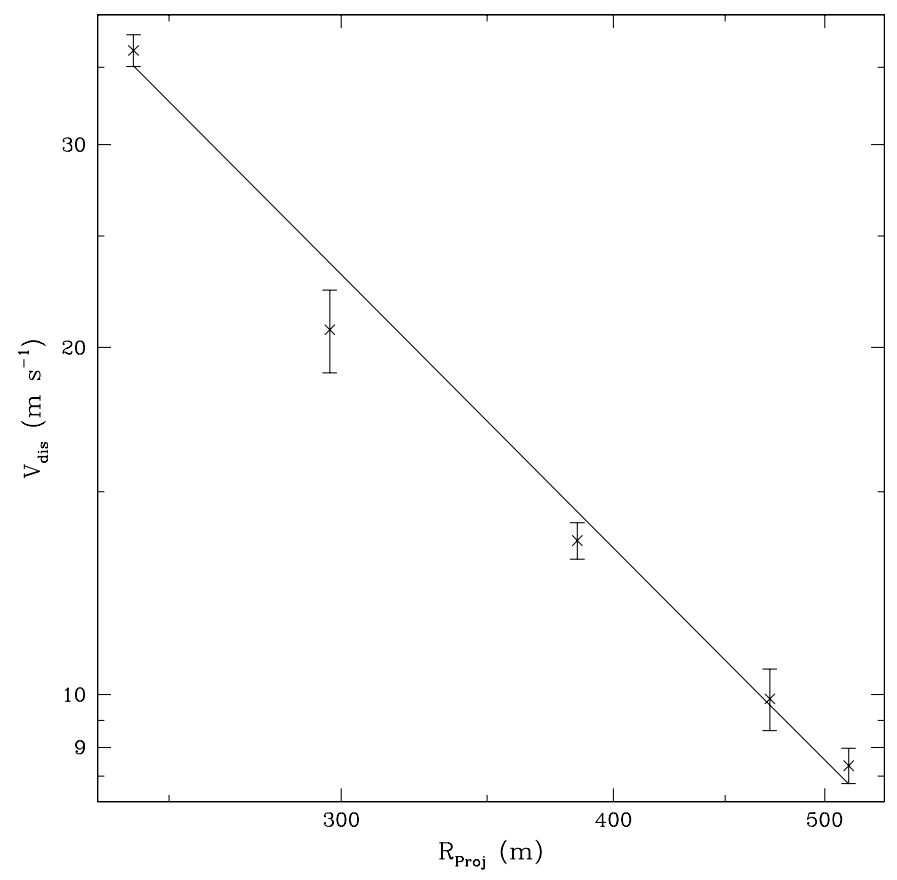

FIG. 6. Critical dispersal speed $V_{\text {dis }}$ as a function of projectile radius. The solid line is a power-law fit with a slope of $-1.9 \pm 0.1$. The error bars represent spread in the critical speed as a result of orientation of the projectile.

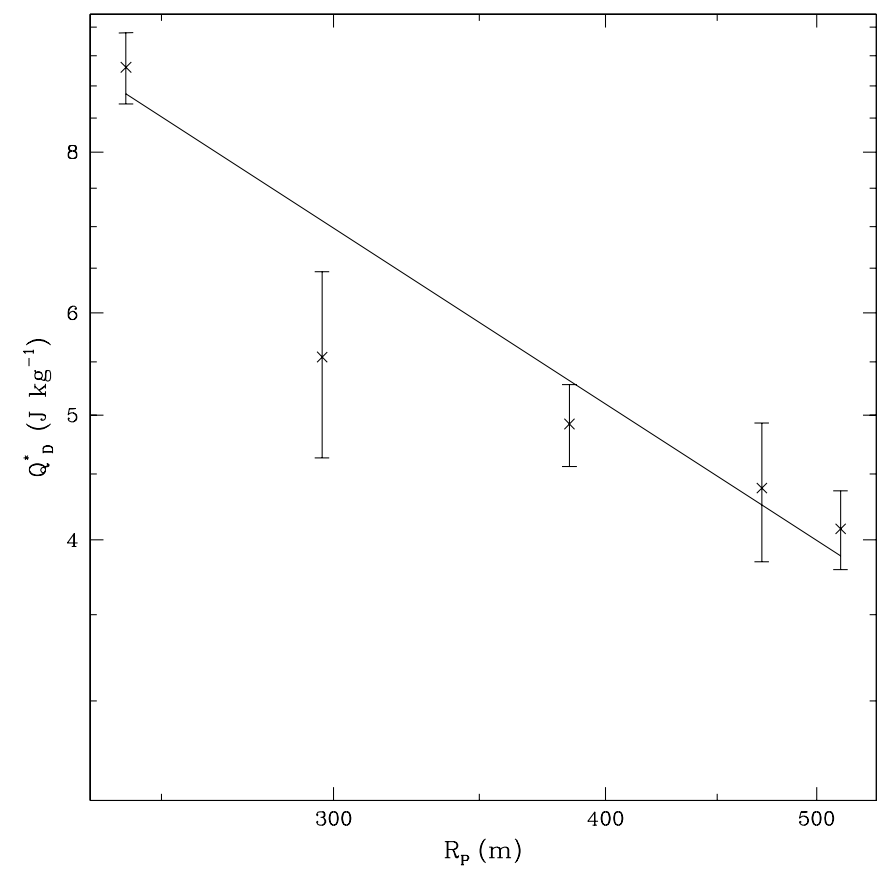

FIG. 7. Critical disruption energy $\left(Q_{D}^{*}\right)$ as a function of projectile radius $\left(R_{P}\right)$. The solid line is a power law fit with a slope of $-1.1 \pm 0.3$ and an intercept of $\log \left(Q_{D}^{*}\right)=3.5 \pm 0.7$.

Ryan and Melosh (1998) used a slightly different method. Using a 2D hydrocode with three different equations of state and including strength and fracturing, they ran a series of simulations to determine $Q_{D}^{*}$ vs $D$ from the strength through the gravity regime by varying the target diameter from $10 \mathrm{~cm}$ to $1000 \mathrm{~km}$. They calibrated their code with impact experiments in the strength regime. However, they did not have a similar calibration for the gravity portion of their code. The impact speed was kept constant at $2 \mathrm{~km} \mathrm{~s}^{-1}$ and the projectile size was varied to find $Q_{D}^{*}$. However, the mass ratio was consistently much more extreme than that of Love and Ahrens (1996); thus the change in the projectile size may not be as important.

\section{CONCLUSIONS}

In this paper we presented results from two sets of direct $\mathrm{N}$-body experiments in order to investigate the collisional evolution of gravity-dominated planetesimals. In these simulations we focused on understanding the effect of impactor mass ratio on collision outcome. In our first set of simulations (Section 3.2) we presented four parameter-space studies, each with a different mass ratio. In these studies we found that as mass ratio increases the impact parameter becomes less important. There was almost no change in $V_{\text {trans }}$ from $b=0$ to $b=0.75$ for mass ratio $1: 6$ and $1: 9$ (Fig. 3). As one might expect the probability of planetesimal growth increases steadily with decreasing $\xi=M_{P} / M_{T}$. For the mean mass ratio $\sim 1 / 5$ (assuming a size distribution $\propto R^{-3}$ ) the probability of an accretion event was $\sim 60 \%$. In 
addition we found that the size of the projectile is important to the collision outcome. A larger projectile is more efficient at disrupting a target than a smaller projectile for the same impact energy.

In the second series of experiments we conducted several head-on simulations at mass ratios far from unity $(1: 8$ to $1: 64)$ in order to find $Q_{D}^{*}$ for a $1-\mathrm{km}$ target. Based on a power-law fit to the above results we found $Q_{D}^{*}=10^{2.8}-10^{4.2} \mathrm{~J} \mathrm{~kg}^{-1}$ for a $1-\mathrm{m}$ projectile.

There are two limitations to our numerical model that must be mentioned. First, because our model does not include a fracture model we are limited to relatively slow speeds. Although this does not affect our current results extensively, we will need to model particle damage in order to extend the speed distribution. Second, all of the simulations presented here were done at relatively low resolution. In order to find out how the detailed mass distribution of the smaller post-collision remnants varies with speed, impact parameter, and mass ratio, higher resolution will be required.

\subsection{Future Work}

The present study had a fairly narrow focus so there are many avenues to explore in future work. We previously mentioned that collision outcome will depend on impactor spin and the choice of dissipation parameter $\epsilon_{n}$-the latter effect in particular remains to be quantified. Ultimately our goal is to implement a planetesimal collision outcome "recipe" in a large-scale planetesimal evolution (planet formation) code, without having to resolve each collision in detail. To achieve this, it will be necessary to parameterize detailed collision simulations by the postcollision fragment/remnant mass and velocity distributions and derive representative distribution functions from these that can be sampled with random deviates. This would not require much more work than the present study and therefore this objective is definitely within reach.

\section{ACKNOWLEDGMENTS}

The authors thank Dr. Erik Asphaug (UCSC) for the use of his Beowulf cluster. They also thank the numerical group at the University of Maryland for their helpful comments. Z.M.L. thanks John Ohlmacher and Chance Reschke for the Borg.

\section{REFERENCES}

Benz, W. 2000. Low velocity collisions and the growth of planetesimals. Space Sci. Rev. 92, 279-294.

Benz, W., and E. Asphaug 1994. Impact simulations with fracture. I. Method and tests. Icarus 107, 98-117.

Benz, W., and E. Asphaug 1999. Catastrophic disruptions revisited. Icarus 142, $5-20$

Durda, D. D., R. Greenberg, and R. Jedicke 1998. Collisional models and scaling laws: A new interpretation of the shape of the main-belt asteroid size distribution. Icarus 135, 431-440.

Holsapple, K. A. 1994. Catastrophic disruptions and cratering of Solar System bodies: A review and new results. Planet. Space Sci. 42, 1067-1078.

Leinhardt, Z. M., D. C. Richardson, and T. Quinn 2000. Direct $N$-body simulations of rubble pile collisions. Icarus 146, 133-151.

Love, S. G., and T. J. Ahrens 1996. Catastrophic impacts on gravity-dominated asteroids. Icarus 124, 141-155.

Richardson, D. C. 1994. Tree code simulations of planetary rings. Mon. Not. R. Astron. Soc. 269, 493-511.

Richardson, D. C., T. Quinn, J. Stadel, and G. Lake 2000. Direct large-scale $\mathrm{N}$-body simulations of planetesimal dynamics. Icarus 143, 45-59.

Richardson, D. C., Z. M. Leinhardt, H. J. Melosh, W. F. Bottke, Jr., and E. Asphaug 2002. Gravitational aggregates: Evidence and evolution. In Asteroids III (W. F. Bottke, Jr., A. Cellino, P. Paolicchi, and R. P. Binzel, Eds.). Univ. of Arizona Press, Tucson. In press.

Ryan, E. V., and H. J. Melosh 1998. Impact fragmentation: From the laboratory to asteroids. Icarus $133,1-24$. 